\title{
Color Flow Imaging in Neurosonology: Technical Background and Clinical Applications
}

Camilo R. Gomez, M.D., ${ }^{*}$ A.F.A.C.A., Asma Q. Fischer, M.D., ${ }^{\dagger}$ and Sandra M. Gomez, M.D. ${ }^{\dagger}$

St. Louis, Missouri, and Augusta, Georgia.

\section{DOI: http://dx.doi.org/10.5915/20-4-13296}

\begin{abstract}
This paper summarizes the technical background and current clinical applications of color flow imaging in neurosonology. The criteria for interpretation along with our experience with color flow imaging of the carotid arteries and the intracranial vessels of neonates is presented.
\end{abstract}

Key words: Color flow imaging, Doppler ultrasound, B-mode ultrasound, carotid bloud flow, neurosonology

The development of duplex ultrasound instruments, which combine high-resolution, real-time, B-mode imaging with pulsed Doppler flow analysis, represented a major step in the non-invasive evaluation of vascular diseases, particularly those affecting the cerebral circulation. The B-mode component of such instrumentation allows the identification of small plaques that fail to cause significant Doppler abnormalities. The Doppler component, on the other hand, is useful in identifying hemodynamic disturbances caused by soft plaques which, owing to their sonolucency, may be missed by B-mode imaging alone. Lately, the concept of duplex scanning has evolved into the more sophisticated technique of color-coded Doppler vascular ultrasonography or, simply, color flow imaging. Originally introduced as angiodynography (angio = vessel; dyno = power, force, or movement; graph = picture, image), it allows the dynamic display of a color-coded Doppler image of the intravascular contents, superimposed on the B-mode, gray scale picture of the vessel. ${ }^{1,2}$ Color flow imaging (CFI) in the neonatal brain can demonstrate the relative direction and velocity of blood flow in color superimposed on a conventional grayscale ultrasound image that depicts stationary tissue of the neonatal brain. Although still not widely available, this technique promises to bring into light new ideas with regards to the various hemodynamic

From the Department of Neurology, The Stroke Resource Center, ${ }^{*}$ Cerebrovascular Service, ${ }^{+\dagger}$ St. Louis University Medical Center, St. Louis, Missouri, and the Department of Neurology, Section for Pediatric Neurology, ${ }^{\dagger}$ Medical College of Georgia, Augusta, Georgia.

Reprint requests: Asma Q. Fischer, M.D., Section of Pediatric Neurology CK 292, Medical College of Georgia, Augusta, GA 30912-3255. effects of different types of vascular diseases in adults and children.

\section{Technical principles}

The basis for color flow imaging is the ability of modern array processing to calculate, at high speed, the amplitude, phase and frequency of ultrasound signals reflecting from the tissue. Slowly moving or stationary tissue signals become part of the real-time image. These signals are processed for amplitude in the same way as those displayed by conventional B-mode imaging. These signals are then assigned a gray-scale intensity proportional to the echo signal amplitude. In this sense, the instrumentation is no different than any other real-time imaging ultrasonic scanner.

Simultaneously however, the phase of the echo signals yields information about the presence and direction of motion (Figure 1a). Changes in phase evoke a color assignment. ${ }^{\prime}$ The two extreme colors, red and blue, can be arbitrarily assigned to represent flow away or towards the transducer. By convention, the RED COLOR, in its different shades, is displayed so it represents arterial flow while the BLUE COLORS are used to show venous flow. The shades of these two colors are graded depending on the velocity based on this principle, lighter colors (either red or blue) represent higher velocities. The lightest of them all, WHITE, will be representative of the maximum velocity associated with the maximum Doppler shift frequency. To allow for a more realistic and dynamic visualization of the color images and their relationship to the cardiac cycle, each pixel is updated approximately 18 times per second in each image frame. When played sequentially, this results in a real-time, color-coded visualization of the intravascular contents. This image, in turn, is representative of the hemodynamic characteristics of flow within the 


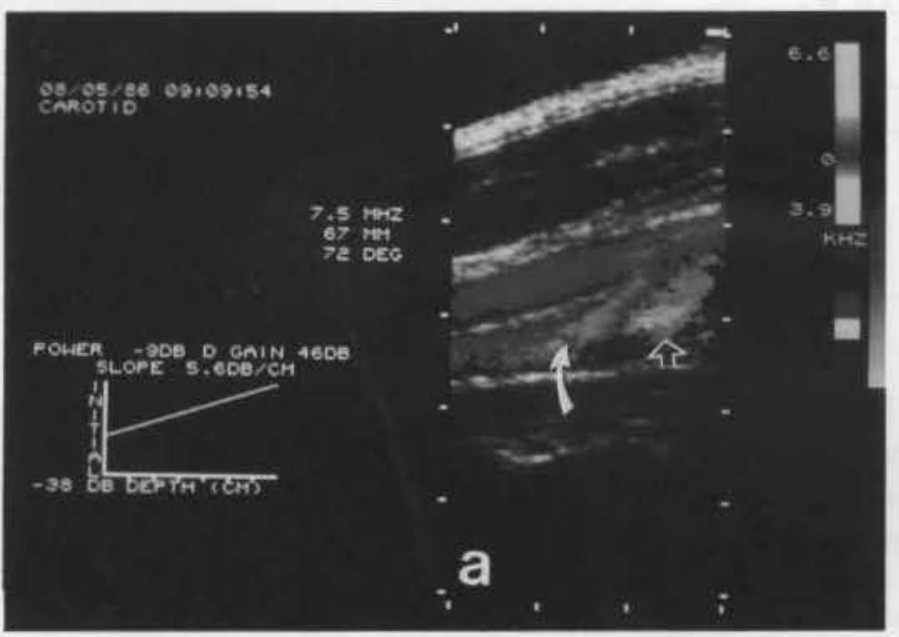

Figure 1a: Normal color flow imaging of the carotid artery bifurcation. Laminar flow is shown as lighter red color in the axis of the vessel lumen (curved arrow). Blue area represents normal flow separation within the carotid bulb (open arrow).

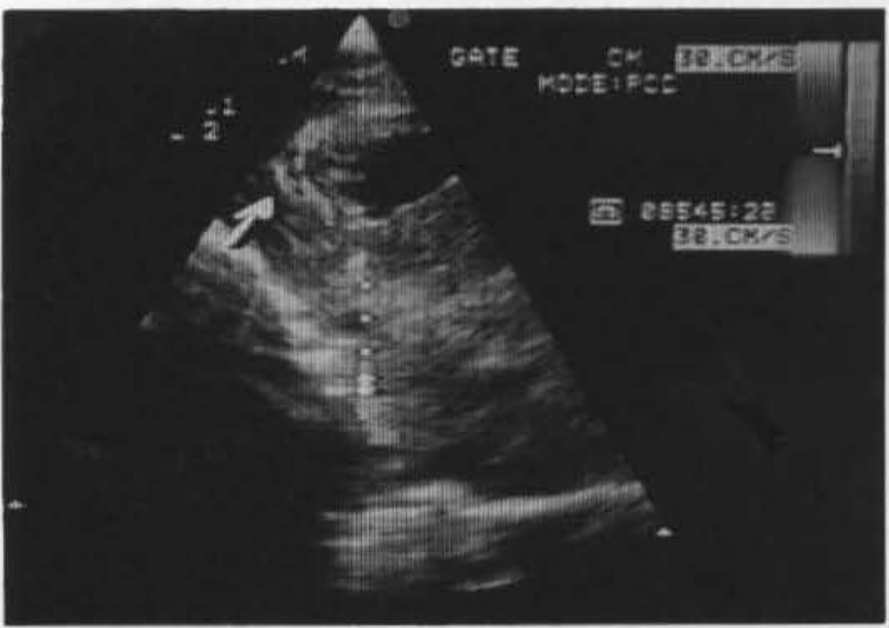

Figure 1c: Mid-line sagittal sonogram of the neonatal brain. The cursor is at the basilar artery as it ascends adjacent to the pons. The white arrow indicates the anterior cerebral artery as it curves around the corpus callosum. The red and blue colors indicate velocity range.

vessel (Figures 1a, 1b). ${ }^{1}$

In CFI of the neonatal brain, the red and blue colors are assigned velocity ranges to assist in finding the highest velocity signals. However when flow direction is being assessed as in patients undergoing

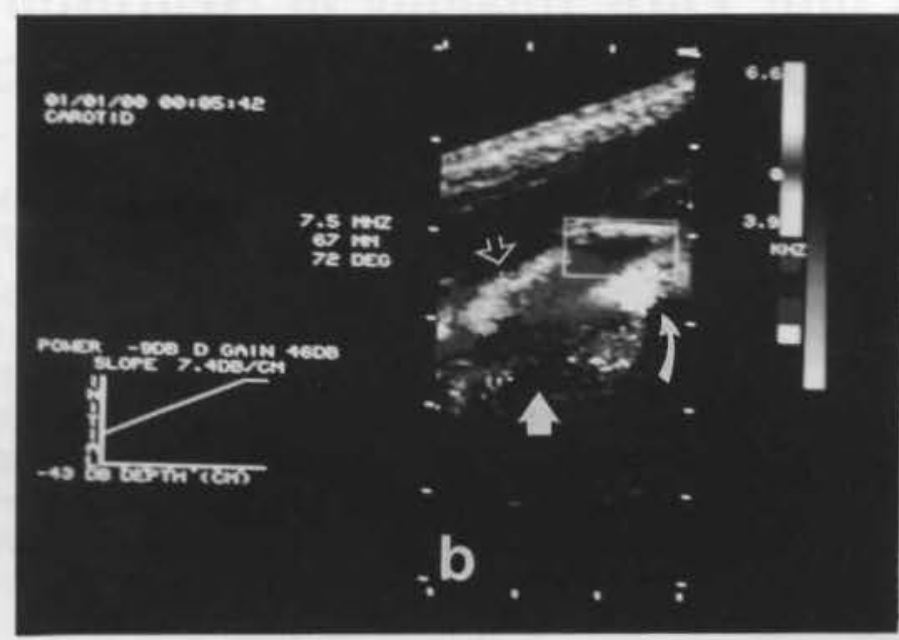

Figure 1b: Color flow imaging of a diseased carotid artery bifurcation. High flow velocity across the area of stenosis is shown in white (curved arrow). Poststenotic turbulence and flow separation shown as mixtures of blue, distal to the stenosis (open arrow). An area of interruption of flow (closed straight arrow) represents artifactual shadow.

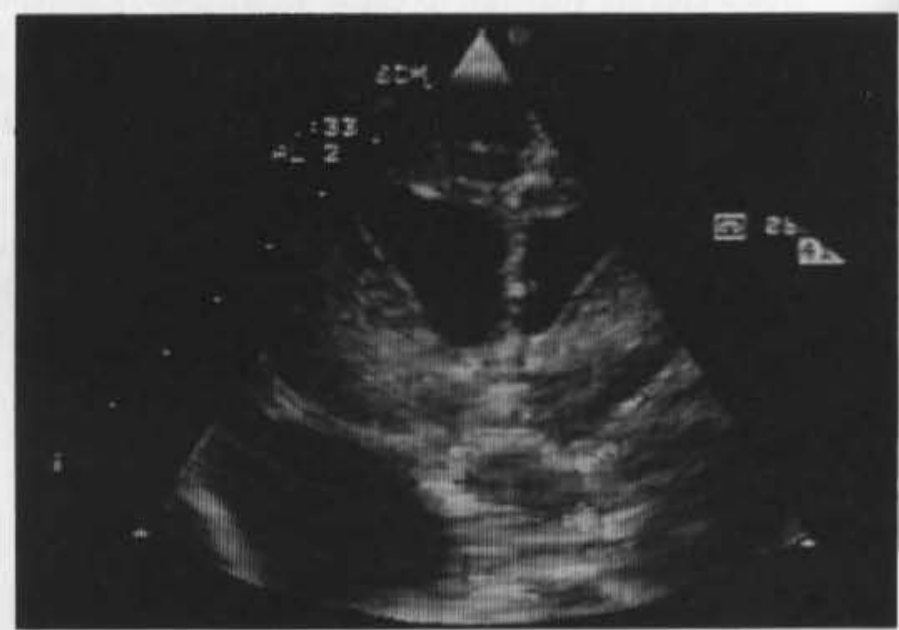

Figure 1d: Rostral coronal sonogram in a hydrocephalic neonate. The two internal carotids are seen ascending and each shows a $\mathrm{Y}$ configuration as it divides into the middle and anterior cental arteries (MCA and ACA).

extra corporeal membranous oxygenation (ECMO), the classic methodology of assigning red to antegrade flow and blue to retrograde flow is used.

Originally, linear array transducers were utilized to perform color flow imaging. Lately, different 
manufacturers have introduced mechanical sector transducers that also yield similar images. Other advances include the introduction of transducers of $3.0-5.0 \mathrm{mHz}$ for the purpose of evaluating deeper structures such as those localized within the abdomen, in addition to the original $7.5 \mathrm{mHz}$ transducers designed for the examination of superficial vessels such as the carotid arteries, and the intracranial arteries in the neonate.

The ideal plane to image and sample the middle cerebral artery (MCA) is through the transtemporal approach. CFI of the anterior cerebral artery (ACA) is done in the mid-sagittal plane (Figure 1c) for the best Doppler signals. CFI of the basilar artery is best done in the mid-sagittal plane (Figure 1c). The anatomy of the intracranial vessels whether normal or distorted by pathology is much easier to identify by CFI than by conventional duplex sonography.

The internal carotid arteries (ICAs) are best seen in the rostral coronal plane as they ascend (Figure 1d) just prior to dividing into the ACA and MCA.

\section{Criteria for interpretation}

The interpretation of color flow imaging is based on simple principles:

1. The gray-scale component of the image should be analyzed in the same way as its counterpart in conventional duplex scanning. The criteria for evaluating plaque morphology (presence or absence of calcification, hemorrhages or ulcers), vessel wall abnormalities, and other aspects of real-time imaging of tissue, are no different in the instruments using color-flow imaging.

2. Although the normal appearance of color flow images is based on the hemodynamic principle of laminar flow, even in normal individuals, variants of this pattern can be seen. Essentially, laminar flow implies that the flow in the axis of the vessel is faster than that close to the vessel wall. The reason for this is that the shear rates are lower in the axial column of flowing blood due to the lack of friction from the vessel wall. When laminar flow is present, the normal color flow image of an artery will display the axial portion of the vessel in lighter colors (i.e. higher velocities) than the portion close to the vessel wall (Figure 1a). Disturbances of this laminar flow, as they will be discussed below, can represent either pathologic conditions or physiologic variants. ${ }^{3,4}$

3. The relationship between the laminar flow pattern and the cardiac cycle is also important in the interpretation of color flow images. This relationship is usually constant but dependent upon the type of vessel being imaged. For example, the internal carotid artery, a vessel supplying a low resistance territory, has significantly higher enddiastolic flow than the external carotid artery which supplies a muscular capillary bed of high resistance. The importance of this dynamic concept cannot be overstressed since lack of diastolic flow in vessels such as the common carotid artery may reflect a significant distal stenosis or occlusion, resulting in stagnation during the lowest point of the cardiac cycle.

4. The relationship between the color flow images and the conventional Doppler ultra sound also has to be assessed. Without this consideration, color-flow imaging yields no more than just "pretty pictures". There has to be consistency and correlation between methods such as spectral analysis of maximum frequencies/velocities, and the color-flow images. Should the two findings not correlate, a critical assumption can be made that one of the two is inaccurate. To make a solid correlation, it is important to compare the portion of the color flow image which directly corresponds with the point of the spectral Doppler sampling.

\section{Clinical applications}

As with any other new technique, especially one which requires the acquisition of expensive instrumentation, significant skepticism surrounds the usefulness of color coded Doppler vascular ultrasound. In spite of this, its popularity has been growing at such a pace that many different manufacturers of ultrasound equipment have already introduced their own version of color flow imaging instruments.

To assess its need each prospective user should ask the question: "What information can be obtained with color flow imaging, that is not available by conventional duplex scanning?" It is our own experience that, in the majority of situations, duplex scanning is sufficiently effective to evaluate most individuals with cerebrovascular disease. There are some instances, however, in which the additional utilization of color flow imaging results in a better understanding of the pathological process in question and its hemodynamic effects. What follows is a description of the two areas of neurosonology where color flow imaging appears to be of marked usefulness.

\section{Color flow imaging of the cervical carotid arteries}

In the field of neurosonology, the evaluation of the common carotid bifurcation in the cervical region is probably the most popular application of color flow imaging. This technique appears to be ideal not only for the differentiation between normal and abnormal conditions but has also provided information that could not otherwise have been obtained. ${ }^{4-6}$ Since the introduction of color flow imaging, the understanding of the effects of anatomical variations of the carotid arteries has grown tremendously. One variant consists of a bulbous dilatation following the bifurcation of the common carotid artery. This dilatation 
is known as the carotid bulb. Its presence has been associated with a very distinctive flow pattern which includes separation and even reversal of the direction of the flow close to the wall of the bulb itself (Figure 1a). ${ }^{2-4}$ This normal pattern, which does not appear to be associated with any symptomatology, had not been graphically demonstrated in vivo in human subjects until now.

In addition to displaying unusual patterns of flow, the presence of eddies, flow reversals, and high velocity, bright white jets associated with significant stenosis, make color flow imaging of the carotids a very powerful method for detecting hemodynamic and emboligenic abnormalities (figure $1 \mathrm{~b})^{5,6}$. The differentiation between all three vessels of the carotid bifurcation (the common, internal, external carotid arteries) becomes very easy with color flow imaging. The flow of the first two is constantly present during the cardiac cycle. The flow of the external carotid artery, on the other hand, stops during diastole. Another advantage for identification is the ability to image branches of the external carotid artery in the cervical region.

We have described the advantage of color flow imaging of the carotid arteries compared with that of conventional duplex scanning: ${ }^{6,7}$

1. Delineation of patterns of flow not previously recognized in both normal and atherosclerotic carotid arteries. As stated above, the graphic demonstration of the asymptomatic occurrence of reversal of flow in normal individuals has provided some basic information about the hemodynamic characteristics of the carotid bifurcation and has raised questions about the role of this flow separation in the production of atherosclerosis.

2. The documentation of patterns of turbulence at the surface of atherosclerotic plaques of unknown hemodynamic significance. Color flow imaging has shown that not all small plaques seem to have the same emboligenic characteristics. Those most commonly associated with ipsilateral cerebral ischemia show surface flow separation which probably represents changes in the electrical charge of the plaque, with subsequent platelet attraction and adhesion.

3. The rapid identification of ulceration in the surface of atherosclerotic plaques.

4. Description of the relationship between the carotid flow abnormalities and the cardiac cycle.

5. Analysis of the effects of carotid artery tortuosity upon the pattern of laminar flow. For years now, it has been an accepted idea that congenital vessel tortuosity is mostly asymptomatic. The identification of hemodynamic abnormalities in relationship to these, will probably allow a more practical classification of carotid artery tortuosities, according to their pathogenic role in symptomatic individuals.

6. More reliable indicator of total occlusion of the carotid artery. The "blind spot" of conventional duplex scanning has been the ability to differentiate between totally occluded carotids and those with $99 \%$ stenosis. Color flow imaging makes this task more dependable.

\section{Intracranial color flow imaging in children:}

Color flow imaging in infants has become an important adjunct of duplex Doppler sonography of the intracranial vessels. The identification of the vessels and direction of the flow can be ascertained with ease." Use of CFI shortens the learning curve for the sonographer and decreases the total time of the study thereby decreasing the exposure time of the neonatal brain to pulsed Doppler ultra sound.' Since the safety of pulsed Doppler ultrasound is of concern ${ }^{10,11}$ and since the ultrasound energy is greater than that with either B-mode imaging, or CFI, it is advantageous to use CFI as it markedly decreases the total time of the study. ${ }^{9}$

The major clinical application of CFI has been in neonates who have been treated with ECMO. ECMO is considered to be a lifesaving procedure for neonates with severely impaired lung function. ECMO requires ligation of the common carotid artery. Bilateral antegrade flow is noted in normal anterior, middle, and posterior cerebral arteries in the neonate' (Figure 1c). CFI has demonstrated altered flow patterns in patients with ECMO. ${ }^{12}$ These patients have retrograde flow in the distal internal carotid artery and the proximal segment of the anterior cerebral artery.

\section{Conclusion}

In this article we have summarized the basic techniques of CFI in neurosonology. We have outlined the current applications and advantages. However, extensive work still needs to be done in the neurosonologic applications to determine areas where CFI is superior to conventional duplex Doppler, before CFI becomes as widely used as Doppler sonography is currently used.

\section{References}

1. Powis RL: Angiodynography: a noninvasive vascular contrast medium. Diag Imaging 1985; $12: 275$.

2. Merritt CRB: Doppler blood flow imaging: integrating flow with tissue data. Diag Imaging 1986; 11:146-155.

3. Ziegler RE, Phillips DJ, Beach KW, et al: Noninvasive assessment of normal carotid bifurcation hemodynamics with color flow ultrasound imaging. Ultrasound Med Biol 1987; 13(8):471-476.

4. Middelton WD, Foley WD and Lawson TL: Flow reversal in the normal carotid bifurcation: 
color doppler flow imaging analysis. Radiology 1988; 167:207-210.

5. Ackroyd N, Laue R, Dart L and Appleberg M. Color-coded carotid Doppler imaging: an angiographic comparison of 324 bifurcation. Aust NZ J Surg 1984; 54(6):509-517.

6. Schwartz RE and Gomez CR: Angiodynography in the evaluation of extracranial carotid artery disease. Proceedings of the VII Panamerican Congress of Neurology. Page 12; 1987.

7. Marritt CRB: Doppler color flow imaging. J Clin Ultasound 1987; 15:591-597.

8. Mitchell DG, Merton D, Needleman Lethal: Neonatal brain: color doppler imaging, Part I. Technique and vascular anatomy. Radiology 1988; 167:303-306.

9. Fischer A Q, Aziz E, Blalock A: Color flow Doppler Sonography of the intracranical arteries (Abstract). Ann Neurol (1988; 24: 344.
10. American Institute of Ultrasound (AIUM): Acoustical data for diagnostic ultrasound equipment, manufacturers commendation panel. Bethesda, MD. AIUM 1985.

11. Taylor KJW: A prudent approach to Doppler us. Radiology 1987; 165:283-284.

12. Mitchell DG, Merton D, Desai Hetal: Neonatal brain: color doppler imaging, Part II. Altered flow patterns from extracorporeal membrance oxygenation. Radiology 1988; 167:307-310.

\section{Acknowledgement}

The authors with to express their appreciation to Miss Teresa M. Palos and Ms. Barbara Harris for their secretarial assistance in the preparation of the manuscript, and to QUANTUM Medical Systems, Inc. for their support in the publication of the color illustrations. 